\title{
QUATTRO CHIACCHIERE A PROPOSITO DELLA COMUNICAZIONE DELLE SCIENZE. LA CHIMICA
}

\author{
Nota del m.e. ANGELO ALBINI (*)
}

(Adunanza del 14 marzo 2019)

SunTO. - La completa separazione tra i linguaggi delle varie scienze rende impossibile comunicare tra specialisti di aree diverse. Il linguaggio della chimica si basa su nomi non ambigui, che mostrano come gli atomi sono disposti e fissati attraverso legami chimici (o covalenti) a formare molecole. Tali nomi suggeriscono in modo euristico le modalità di sintesi. Inoltre le equazioni chimiche indicano il meccanismo che porta da una situazione iniziale (reagenti) ad una finale (prodotti). In molecole molto grandi (etero ed omo polimeri) il legame chimico non è sufficiente a illustrarne la reale struttura, ma la conformazione nello spazio è in realtà già dettata dal modo in cui gli atomi sono legati. La risposta delle molecole a variazioni nell'ambiente sono adatte a un universo che si trasforma con passaggi dolci, non a scatti.

$* * *$

ABSTRACT. - The language used within different disciplines has become fully separated. The language of chemistry is based on chemical names, where it is shown how atoms are disposed through chemical or covalent bonds to yield molecules, On the other hand, assigning a name contains as an added bonus the indication of which heuristic way/ways are suitable to prepare them that to transform an initial situation (reagents) to a final one (Products). In big molecules (homo and hetero polymers) chemical bonds are not sufficient per se to describe molecules, but the spatial conformations such molecules take are dictated by the bonds structure. The way molecules change answering a change in the environment are appropriate for a universe changing though smooth steps, not via shots.

(") Emerito presso il Dipartimento di Chimica dell'Università di Pavia, Italy. E-mail: angelo.albini@unipv.it 


\section{NOMI CHIMICI}

Oggi non ha senso parlare di scienza, intesa come un unico corpo organizzato di conoscenze, ma semmai di scienze, discipline diverse che scorrono su strade parallele e ricorrono ciascuna a uno specifico linguaggio. Da sempre gli umani hanno osservato l'ambiente nel quale vivono, notando alcune correlazioni fra un dato evento e un dato effetto, ad esempio il costante avvicendamento delle stagioni e il mutare delle temperature, o le scottature che seguono una lunga esposizione al sole. Erano fenomeni osservati, ma non spiegati. Con lo sviluppo della scienza occidentale, è stato possibile spiegare il perché di tali fenomeni e, quando da un dato evento risulta una malattia, si è cercato di proporre un possibile rimedio.

Purtroppo il linguaggio delle scienze, o meglio, il modo in cui ciascuna di esse racconta il proprio mondo, è complicato dal fatto che ognuna di esse ha un suo codice di riferimento, e non è affatto semplice 'tradurre' le cose dall'una all'altra (nelle numerose occasioni in cui le varie scienze si intrecciano), anche quando le parole sembrano le stesse. Scienziati di diverse discipline declinano segni e fonemi in maniera diversa.

Una particolare possibile descrizione dell'universo è quella chimica, che si basa sulla comunicazione di come siano legati gli atomi nelle molecole che costituiscono il mondo intero. Nel seguito si presentano tre aspetti generali del linguaggio della chimica e della loro relazione con la prima e la più importante (oppure la meno importante in quanto meno razionalizzata) tra le scienze, a seconda del punto di vista scelto), la medicina). È sempre stata un'aspirazione della chimica quella di avere un siste$\mathrm{ma}$ in grado di assegnare un nome non ambiguo alle sostanze.

Nel clima ottimista della Belle Èpoque, in cui il progresso stava per raggiungere l'acme delle scoperte, si sviluppò l'idea che si potesse costituire una enciclopedia chimica di base con una struttura non più da variare, ma da aggiornare quando fosse il caso con nuovi dati da inserire nella griglia. Questa idea fu realizzata con il Beilstein Handbuch der organische Chemie,[1,2] la cui prima edizione fu pubblicata nel 1881 e universalmente considerata un contributo monumentale alla chimica del popolo tedesco (Fig. 1). Purtroppo, la grande guerra distrusse la fiducia nel progresso e bloccò lo sviluppo del piano di aggiornamento dell'opera, ma terminata anche la seconda guerra mondiale, il progetto ripartì e, al giorno d'oggi, è disponibile anche in una raffinata forma informatica. 


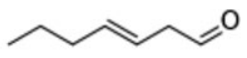

E-3-eptenale<smiles>CC1=CC(C(=O)N(C)C)CCC1</smiles>

3, $\alpha, \alpha$-trimetilcicloesencarbossiamide $\quad N, N$-ciclopropilacerammide<smiles>CN(C)C(=O)C1CC1</smiles>

Fig. 1. Esempi di nomi chimici.

e regredendo man mano verso composi più semplici. Lo stesso vale, naturalmente, per la chimica inorganica, in cui la varietà dipende soprattutto dalle differenti possibilità che vi sono per i vari elementi di partecipare alla formazione di molecole o ioni con diversa valenza (Gmelin Handbook of Inorganic and Organometallic Chemistry).[3] Vedi Fig. 2,3,4.

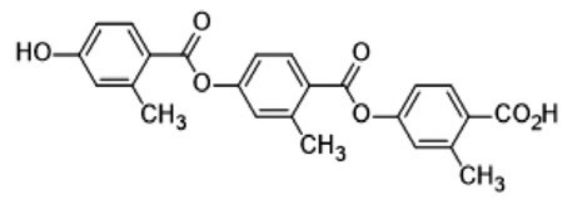

Fig. 2. Esempi di nomenclatura non sistematica, depsidi (a sinistra) e depsidone (a destra).
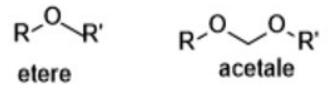

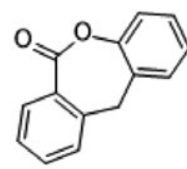

Fig. 3. Nomenclatura sistematica applicata a molecole con numeri crescenti di legami C-O

$\begin{array}{ccccc}\mathrm{Cl} & \mathrm{Cl}+{ }^{+} & \mathrm{Cl}^{+} \mathrm{O}_{2} & \mathrm{Cl}^{+5} \mathrm{O}_{3} & \mathrm{Cl}^{+7} \mathrm{O}_{4} \\ \text { anioni cloruro } & \text { ipoclorito } & \text { clorito } & \text { clorato } & \text { perclorato } \\ & \mathrm{S}^{4+} \mathrm{O}_{3}^{2-} & \mathrm{S}^{6+} \mathrm{O}_{4}^{2-} & \mathrm{S}^{8+} \mathrm{O}_{5}^{2-} & \\ \text { solfito } & \text { solfato } & \text { persolfato }\end{array}$

Fig. 4. Esempio di atomi che partecipano alla struttura di anioni con differente valenza. 
Naturalmente, queste nozioni di stechiometria, per essere verificate, non hanno alcun bisogno di quantomeccanica, pur essendo perfettamente inquadrabile in essa; per accertare la composizione atomica è sufficiente una bilancia (omnia mensura sunt, sta scritto in lettere grandi sulle pareti dell'aula magna di chimica a Bologna).

Un paio di esempi potranno aiutare a capire quanto una nomenclatura strutturata possa essere di aiuto. Una delle rarissime volte in cui l'autore di questa nota sentì il professor Grünanger, Direttore dell'Istituto di chimica organica a Pavia, alzare la voce con uno studente durante un esame, fu perché l'esaminando non seppe individuare un gruppo funzionale acetalico nella molecola di cui si discuteva, e cercava di uscire dall'impaccio in cui si era cacciato chiamandola doppio etere, mentre il fatto che vi siano due legami C-O invece di uno solo, come avviene con gli eteri, non porta certo a un 'raddoppiamento' della somiglianza nelle proprietà fisiche e chimiche tra $\mathrm{i}$ acetali ed eteri. D'altra parte, mi piace ricordare un aneddoto che ho sentito più volte raccontare dal professor Silvio Pietra, che ricordava come durante una sessione di esame cui partecipava accanto al suo capo, questi aveva chiesto a uno studente cosa fossero i depsidi, ottenendo una scena muta. Nel corso dell'esame, era emerso che quello stesso studente nulla sapesse di cosa fossero i depsidoni, e la cosa non avrebbe dovuto meravigliare l'esminatore, perchè i due gruppi di composti presentavano solo una somiglianza nei nomi, mentre solo se si usa la nomenclatura sistematica, viene evidenziata ogni possibile differenza di struttura e di sintesi (Fig. 2).

Per avere un'idea più chiara della struttura delle molecole ci si può avvalere della spettroscopia che, grazie all'azione della luce UV e visibile assorbita (o non assorbita) dalle molecole, rivela esattamente come siano fatti i legami. Per questo l'interazione luce-molecole ha un ruolo speciale nella comprensione del perché le molecole, e in particolare le biomolecole, sono così stabili e come reagiscono, aiutando a chiarire meccanismi fondamentali di reazione e anche l'effetto sulle biomolecole e quindi sulla salute.[4] ${ }^{1}$

1 Ad esempio, nel caso della pelle, il maggiore degli organi umani per peso ed estensione, la natura ha predisposto un apposito meccanismo che limita i danni provocati dalla luce sul DNA (che comprendono alcune delle più pericolose forme di tumore, come il melanoma, particolarmente temuta, perché si manifesta a decenni dall'evento che lo ha originato), filtrando la luce a corta lunghezza d'onda. La ricerca scientifica sostituisce la formazione di melanine con filtri artificiali più efficienti. E' ormai noto a 
D'altra parte è necessario che la pelle assorba sufficiente luce perché venga formata un'adeguata quantità di previtamina D (vedi Fig. 5), la cui conversione in vitamina verrà poi completata con ulteriore idrossilazione nei reni e nel fegato. Della vitamina D si sa molto,[5] ma non tutto, e vengono costantemente scoperti nuovi ruoli, oltre al ben noto governo dell'omeostasi del calcio, di ovvia importanza, dato che l'intero scheletro viene rinnovato ogni pochi giorni.[5]
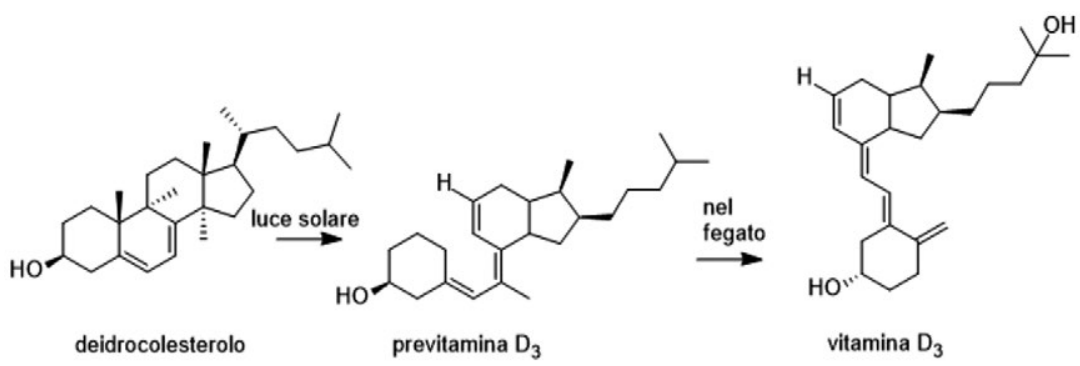

Fig. 5. Formazione della previtamina $D_{3}$ attraverso reazione ettrociclica a 6 elettroni.

I gravi effetti dell'insufficiente assorbimento di luce erano già noti a Erodoto, che scrive di essersi recato sui campi di battaglia e di aver notato che i crani dei guerrieri egiziani erano assai più resistenti di quelli dei nemici, un fatto attribuito al costume egiziano di stare a capo rasato a partire dall'infanzia. E' ormai assodato che l'insufficienza di vitamina $\mathrm{D}$ produce il rachitismo dei bambini e la osteomalacia, il morbo corrispondente degli adulti, detta anche Morbum Anglorum per la stretta correlazione tra le pessime condizioni igieniche caratteristiche della rivoluzione industriale in Inghilterra e questa carenza.[5,6]

D'altra parte il ricorso eccessivo ai lettini UVA per ottenere un abbronzatura costante, può causare un precoce invecchiamento della pelle o l'alterazione del controllo del livello di altre vitamine, in particolare quelle che intervengono su processi ossido-riduttivi, come le vitamine $\mathrm{C}, \mathrm{E}, \mathrm{K}$.

tutti che, specie per persone di complessione molto chiara, è consigliato utilizzare una sufficiente quantità di crema solare, di cui bisogna controllare, più che il fattore di stabilità (che comunque non ha nessun effetto andare oltre il Sun Protecting Factor (SPF) di 30), mentre è fondamentale usarle in condizioni ottimali e riapplicarla spesso (il SPF si riferisce a una quantità tra 0.5 e $1 \mathrm{mg}$ di principio attivo per $\mathrm{cm}^{2}$, purché la si riapplichi abbastanza frequentemente e non si lascino parti del corpo scoperte). 
Sono stati osservati numerosi altri effetti dell'esposizione alla luce solare, per fortuna meno correlati con la salute, ad esempio lo scoloramento e la perdita di robustezza di tessuti naturali e artificiali, tipicamente legate a processi radicalici innescati dalla luce. Tale meccanismo può manifestarsi anche dopo una fase di iniziazione in cui si formano prodotti che assorbono una parte maggiore di luce e sono buoni iniziatori della degradazione, tipicamente i chetoni).

\section{NOMI CHIMICI. CONFORMAZIONI E STRUTTURE TRIDIMENSIONALI}

La considerazione dei legami chimici e dei nomi sistematici dei composti non è tuttavia sufficiente ad esprimere la struttura di molecole più grandi. Consideriamo, ad esempio, le proteine, gli eteropolimeri che si formano dalla policondensazione degli aminoacidi secondo le istruzioni depositate nel DNA. In effetti,

la struttura primaria di una proteina, cioè la sequenza lineare di amminoacidi che la compongono, è sufficiente per giudicarne la composizione, ma non il suo ruolo fisiologico. Questa informazione è tuttavia già presente nella struttura primaria, attraverso la conformazione tridimensionale della proteina nativa. Ogni sequenza polipeptidica e la posizione di ogni residuo aminoacidico nella catena sa come orientarsi nello spazio e come arrivare spontaneamente alla sistemazione preferita o nativa (con notevole crescita di entropia). Il raggiungimento di quella particolare conformazione dipende anche dall'ambiente in cui il processo di piegatura (folding) si completa, ad esempio con la formazione di legami a idrogeno intra e intermoleculari, o in presenza di forze di van der Waals o anche se, ad esempio, il folding inizi o no in maniera co-translationale, cioè mente ancora nuovi legami peptici vengono formati, dalla concentrazione di ioni, dal $\mathrm{pH}$, dalla temperatura, dall'ambiente idrofilo o idrofobo e così via.[7]

Qualcosa di concettualmente simile avviene nella formazione di complessi organometallici a struttura specifica intorno a uno ione metallico. Sia il raggiungimento spontaneo della conformazione nativa che la formazione selettiva di complessi organometallici illustrano come la chimica sia la scienza dell'instabile, del provvisorio, di quanto di più lontano sia pensabile da Goethe e dai tedeschi in genere, nonostante la evidente affinità di quel popolo per tale scienza, e sia dunque l'unica possibile base per la vita, in un certo senso all'opposto della matemati- 
ca, o della (quanto)meccanica, il sempre vero, l'assoluto, l'eterno, il das ewig-weibliche zieht uns hinan del Faust.

Le figure qui inserite danno un'idea della formazione di fogli antiparalleli per legami $(\beta)$ ad idrogeno con ciò formando una struttura secondaria. (vedi Fig. 6)

\section{La struttura secondaria delle proteine}

\section{Struttura secondaria}

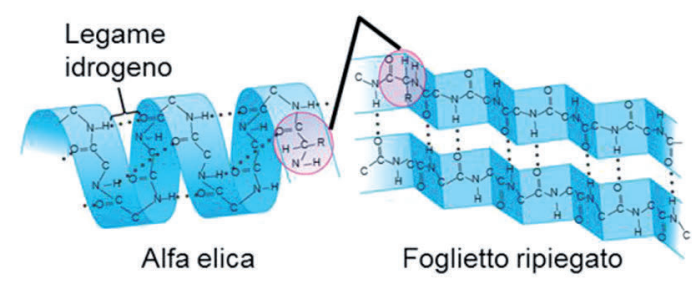

Fig. 6. Self assembing di una proteina, strutture secondarie (da SlidePlayer.it).

Le strutture secondarie offrono il primo passo in questa direzione. Tipiche delle strutture secondarie sono i fogli $\alpha$ e $\beta$, che si avvolgono rapidamente in quanto stabilizzati dalla formazione di legami a idrogeno intramolecolari. Per quanto riguarda le strutture terziarie, queste derivano dalla stabilizzazione tra fogli di natura anfipatica, cioè quelli che contengono una parte idrofila e una idrofoba, e si avvolgono in maniera da presentare la regione idrofila verso l'ambiente che circonda la proteina. Un contributo lo possono dare anche legami covalenti relativamente deboli, come i legami S-S della cistina. Infine, strutture quaternarie possono formarsi in certe proteine attraverso la sistemazione o risistemazione di subunità pre-esistenti.

Ad esempio la sequela di eventi che alla fine produce la contrazione di specifiche fibre muscolari comincia con un segnale, un neurotrasmettitore, ACh fornito dal neurone motore che innerva quella fibra attraverso un complesso meccanismo. Fondamentale per la definizione delle conformazioni pensabili è la rigidità del gruppo ammidico che pone limiti agli angoli formati dalle proteine, come si può valutare attraverso l'indice di Ramachandran.[8] Si può controllare sperimentalente che prodotti di denaturazione esposti ad alcuni fattori denaturanti si ripiegano di nuovo e prendono l'esatta conformazione nativa).[7-9] 
Una proteina completamente denaturata non ha più struttura né secondaria, né terziaria ed esiste nella cosiddetta catena a caso (random coil). In alcuni casi, le cellule proteggono le loro proteine contro l'effetto denaturante del calore co enzimi detti proteine conto i colpi di calore (proteine 'heat shock', un tipo di molecole accompagnatrici),[9] che assistono altre proteine nel processo di folding, nonché nel rimanere poi nella conformazione preferita. Va tenuto presente che non necessariamente le proteine assumono una struttura denaturano, specie se la trasformazione avviene al di fuori delle condizioni di lavoro tipiche (ad esempio questo è motivo per cui le albumine che costituiscono il bianco d'uovo passino ad opaco). La stabilità termica delle proteine è per altro molto varia e son stati trovati batteri ipertermofili che funzionano perfettamente a $122^{\circ} \mathrm{C}$.

Naturalmente, vi sono diverse malattie che dipendono da errori nella sequenza di aminoacidi o da errori nel folding di proteine, in particolare malattie croniche neurodegenerative dovuti al fatto che questi errori rendono poi le molecole incapaci di tornare alla normale struttura nativa. Le proteine indicate come con errori (misfolded), tipicamente contenenti fogli $\beta$ organizzati in maniera supramoleculare, note come fogli- $\beta$ reticolati, assai stabili, del tutto insolubili e molto resistenti all'idrolisi in condizioni acide (strutture amiloidi) ne sono un tipico esempio. La stabilità strutturale di queste componenti fibrillari ne favorisce l'accumulo, eventualmente con altre proteine in aggregati non più in grado di svolgere le previste funzioni fisiologiche. Queste proteine sono collegabili con i morbi di Huntington, di Parkinson e di Alzheimer che tutti esibiscono un accumulo di proteine con errori in aggregati insolubili extra o intra-cellulari (amiloidi); che poi sia questa la causa di tali malattia o invece una conseguenza della perdita di omeostasi di alcune proteine non sembra per ora definitivamente provato.

Gli agenti denaturanti comprendono sia alcune molecole o ioni (come l'urea, il cloridrato di guanidinio, il pH), come anche fenomeni fisici, come temperatura, pressione, e così via. L'equilibrio tra conformazione differenti e diverse $\mathrm{d}$ una proteina dipendono dallo stato nativo, gli stati intermedi non piegati a loro volta dipendono dal denaturante usato, e, con valutazioni attente, si può comprendere quale sia il profilo energetico del processo di folding e unfoldinge e identificare gli intermedi che si formano sul percorso seguito, nonchè ricavare i parametri termodinamici relativi. Va ricordato il numero elevatissimo di conformazioni da confrontare (dell'ordine di $3^{300}$, cioè $10^{143}$ ) che sem- 
brerebbe difficile da raggiugere concretamente se si dovesse valutare singolarmente ciascuna conformazione.[10]

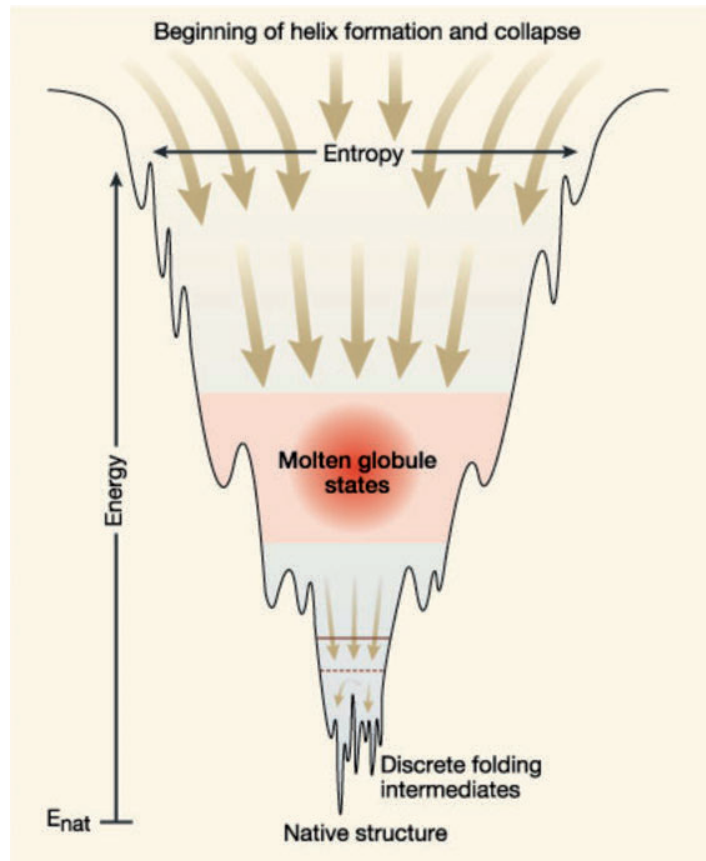

Fig. 7. Panorama degli spostameti nello spazio in funzione del livello energia quando il folding di una proteina e imbuto (funnel) energetico attraverso il quale un polipeptide arriva alla struttura nativa. (da Summary of the Horizon Symposium on 'Protein Misfolding and Disease'Christopher M. Dobson).

Secondo J Bryngelson and P. Wolynes,[11] le proteine seguono il principio di minima frustrazione, nel senso che le proteine come si sono evolute in natura devono aver minimizzato la loro energia, come indicato nella figura. Peraltro qualche grado di frustrazione rimane nonostante l'evoluzione, come indicato dall'esistenza minimi locali nel panorama energetico (vedi Fig. 7).[12]

Di fatto, il raggiungimento della conformazione preferita nelle proteine è stato stimato avvenga attraverso una molteplicità di imbuti, la maggioranza dei quali sono diretti verso lo stato nativo (folding funnel), e non attaverso un solo meccanismo. in tempi che vanno da100 ns a qualche ms, come tipico per i $\beta$ turns. 
Lo stato di transizione, per il quale ogni proteina deve passare per raggiugere la struttura nativa è sostanzialmente una forma non ancora completata di quella finale, non un altro intermedio, e determina la velocità, quandanche vi possano essere altri stati a maggior energia. $[13,14]$

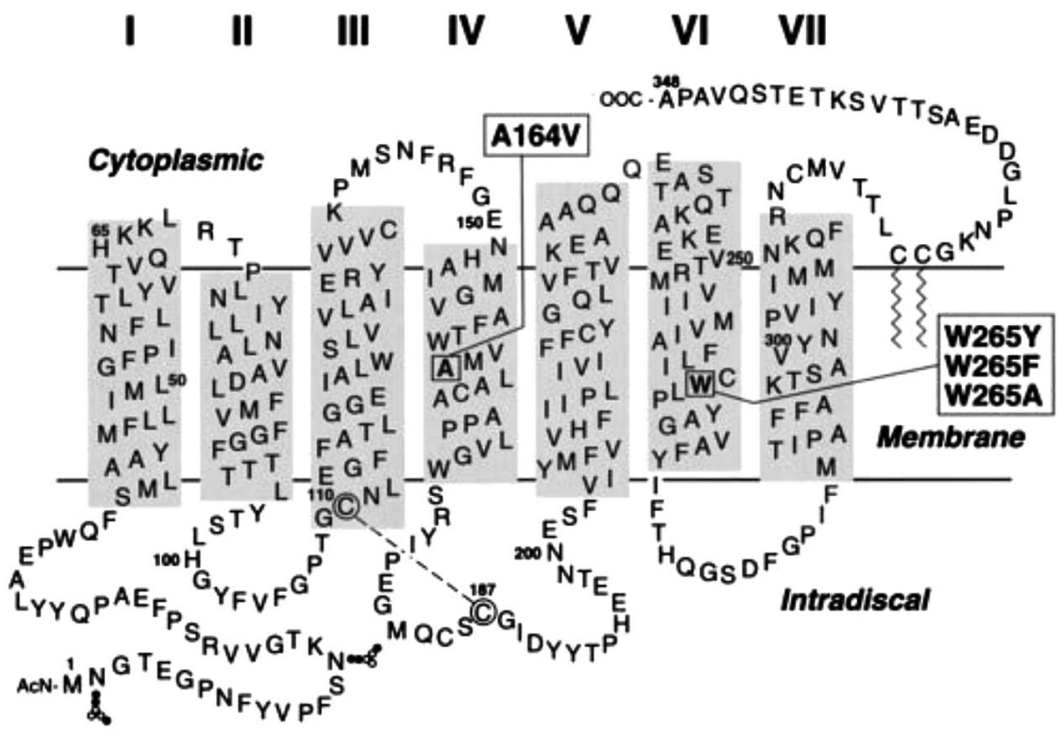

Fig. 8. Struttura bidimensionale della rodopsina. Come d'uso, i singoli amminoacidi sono indicati da una sola lettera. [15]

Nel caso della visione, basata sul ruolo della rodopsina (vedi Fig. 8), il pigmento porpora fotosensibile, che è uno solo anche se la struttura tridimensionale ne consente tre conformazioni differenti, che comportano differenti assorbimenti nello spetto visibile. Il processo fotochimico si deve alla transizione trans cis di un doppio legame $\mathrm{C}=\mathrm{C}$, da 11-trans a 11-cis nella molecola del retinale che è il gruppo prostetico, seguito da una serie di processi termici e infine del distacco del retinale, che viene poi rigenerato attraverso il corrispondente alcool. Le molecole di opsina, una proteina relativamente semplice (poche centinaia di residui amminoacidici) si sistemano in modo da formare sette canali attraverso la membrana che separa il citoplasma dalla soluzione esterna. La drastica variazione nella geometria della molecola di retinale (che è legata con un legame covalente come gruppo immonio, $>\mathrm{C}=\mathrm{N}^{+} \mathrm{R}$ sposta 
notevolmente la sesta catena, e come risultato cambia il passaggio attraverso la barriera, e quindi la concentrazione finale degli ioni $\mathrm{Na}^{+} \mathrm{e} \mathrm{Ca}^{2+}$ (si vedano le figure 8,9 ). Non è questa la sede adatta per discutere la maniera assai complessa che porta alla visione tricromatica (rosso-bluverde come spetro di sensibilità, tonalità, saturazione e luminosità, dal punto di vista quantitativo) degli umani. Per altro non è facile nemmeno pensare a come vedano animali che lo fanno un minor o maggior numero di dimensioni (ad esempio cani, cavalli e squali hanno una dimensione in meno, ma uccelli e pesci una in più, e alcune farfalle molte di più). È da notare però che tutti i vertebrati condividono lo stesso sistema di base con i sette canali della trans-membrana, che è perfezionata ed è rimasta sostanzialmente la stessa da gran tempo in tutte le specie dei vertebrati, molto di più di altre caratteristiche molto importanti, quali la mascella mobile, a indicazione che l'evoluzione si ferma quando una soluzione sodisfacente è stata trovata.

\section{RUOLO DEL RASOIO DI OCCAM IN CHIMICA}

Il concetto stesso di ipotesi scientifiche verificabili va considerato tuttavia con cautela. Ad esempio, il principio della minima azione e minimo spostamento domina la chimica e la scienza in genere, anche se non sempre in maniera consapevole. Si accetta semplicemente il valore euristico del rasoio di Occam, il cui fondamento comporta l'assunzione che il mondo sia semplice, cosa che non è però scontata. Alcuni aspetti di questo problema vengono accennati nel seguito, sulla base di una pubblicazione di R. Hofmann et al.[16]

Il rasoio di Occam può essere espresso con le seguenti affermazioni.

A Frustra fit per plura quod potest fieri per pauciora.

B Quando propositio verificatur pro rebus, si duae res sufficiunt ad eius veritatem, superfluum est ponere tertiam

C Pluralitas non est ponenda sine necessitate.

D Nulla pluralitas est ponenda nisi per rationem vel experientiam vel auctoritatem illius, qui non potest falli nec errare, potest convinci.

Si tratta, dunque, di stabilire la validità di singole ipotesi scientifiche, per quanto l'affermazione D possa servire a giustificare la reintroduzione della pluralità, pur entro limitazioni precise (Nec intra ulla pluralitas est ponenda nisi per rationem vel experientiam vel auctoritatem illius, qui non potest falli nec errare, potest convinci). 
Se s'intende il termine 'meccanismo di reazione' come la descrizione di tutti i passaggi 'elementari' che portano dai reagenti ai prodotti al livello molecolare che quindi include principio almeno, la conoscenza della geometria e dell'energia relativa di tutte le strutture che hanno un ruolo, comprese quelle isolabili, almeno potenzialmente, con intermedi e stati di transizione, e i secondi rappresentanti i punti in cui l'evoluzione di un avanzamento della traiettoria della energia minima che congiungono le specie che si interconvertono. In una definizione alternativa, il meccanismo stabilisce l'evoluzione di un sistema chimico lungo la traiettoria di reazione, attraverso un cammino che congiunge $\mathrm{i}$ reagenti ai prodotti nello spazio di tutte le coordinate nucleari richiede l'introduzione del concetto di superfici dell'energia potenziale (PES). Di fatto, ben prima che l'idea di molecola prendesse piede, il principio di minima azione era già stato sviluppato dopo esser stato introdotto da Pierre Louis Morveau de Maupertuis e applicato universalmente da Leonardo Eulero in balistica, nel movimento di una forza centrale. Secondo questo principio, i moti spontanei avvengono sempre con la minima quantità di 'azione', e questa è una ben definita variabile fisica. In una presentazione svolta a Parigi nel 1744 su questo principio, de Maupertuis sottolineò che ad esempio la luce non segue linea più breve, né la più veloce, ma quella che è più economica (si confronti la legge della parsimonia), cioè quella in cui la quantità di 'azione' è minima.

Non sorprende quindi che, quando negli anni Trenta il meccanismo delle reazioni chimiche diventò più importante, anzi divenne un punto focale della chimica fisica organica, gli studi avvennero con lo spirito ed il vocabolario della meccanica. Peraltro, il primo passo in questa direzione era stato compiuto anche prima, in effetti da Muller nel 1886,[17] cioè in un tempo in cui la teoria molecolare era ancora recente e questo scienziato introdusse la regola della minor variazione possibile nel corso delle reazioni chimiche.[18]

L'idea era attraente e trovò posto in vari libri di testo,[19] ove venne indicato come il principio del minor variazione possibile e fu formulato nei termini più generali da F. Rice e E. Teller, che nel 1938 proposero il principio del minimo movimento (PLM),[20] secondo il quale verranno favoriti i passaggi 'elementari' che riguardano le variazioni minori nella configurazione degli atomi e nella configurazione elettronica'. In questo contesto di grande importanza furono le regole di simmetria degli orbitali molecolari (MOs) che furono introdotte nella chimica organica 27 anni dopo.[21] 
Per applicare il PLM a una certa reazione, gli atomi che costituiscono reagenti e prodotti devono esser spostati l'uno rispetto all'altro in modo che i movimenti dei nuclei (di solito misurati dai loro quadrati) vengano minimizzati. Di fatto, un buon numero di riarrangiamenti, decomposizioni ed eliminazioni sono state dimostrate avvenire attraverso cammini che ubbidiscono alle richieste del PML. La grande semplicità dei calcoli richiesti e, cosa più importante, la chiarezza dell'idea provocarono l'ampia applicazione del PML, in particolare quando bisognava scegliere tra varie alternative. Tuttavia era sempre chiaro a chi usava questo metodo che il PLM rappresentava un modello teorico estremamente semplificato del movimento reale dei nuclei e degli elettroni nel corso di una reazione, un movimento che per esser descritto in maniera adeguata abbisogna della soluzione delle equazioni quantomeccaniche e questo lascia sempre il dubbio che la quantizzazione degli stati elettronici, vibrazionali e rotazionali cambi il risultato. Inoltre, bisogna tener in conto il fatto che è necessario un approccio dinamico per descrivere la reazione come avviene in realtà, cioè attraverso una miriade di percorsi accessibili. Tuttavia il PML va incontro al bisogno generale di semplicità, e quindi il fatto che l'allontanamento dal modello o anche l'incompatibilità con le previsioni del PML non resero mai questo principio un metodo per accettare definitivamente un meccanismo.

Consideriamo per primo una classica reazione periciclica,[22] il riarrangiamento di Cope (spostamento sigmtropico 3,3, Fig. 9). In questo caso, anche una minima variazione nella struttura nell'idrocarburo di partenza, 1'1,5-esadiene, sembra causare una variazione dal cammino più tipico (a) con il suo intermedio a struttura 'aromatica' (in due forme isomere), oppure attraverso i cammini (b) o (c), che son caratterizzati, rispettivamente, da uno stato di transizione biradicalico come stato di transizione o come vero e proprio stato intermedio.[23]
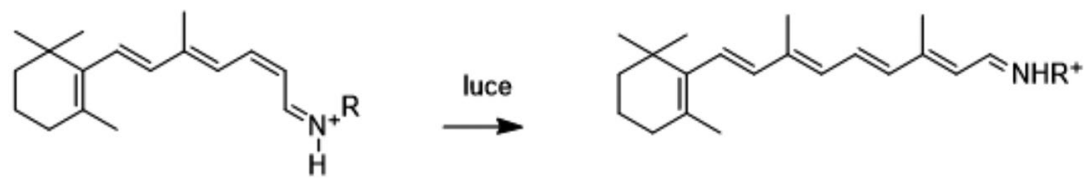

Fig. 9. Tre possibili meccanismi per il riarrangiamento di Cope.

Come esempio, consideriamo quello di fullereni, i prodotti poliedrici ottenuti dalla grafite per vaporizzazione a temperature del plasma $\left(>3000^{\circ} \mathrm{C}\right)$. Contrariamente a una previsione 'entropica' di forme poco 
organizzate strutturalmente, forme della materia altamente organizzate sono la maggioranza, nello specifico molecole altamente simmetriche con poliedri $\mathrm{C}_{2 \mathrm{n}}$ con una struttura che ricorda le cupole geodesiche usate in architettura da R. Buckminster Fuller.[24] La configurazine $\mathrm{C}_{60}$, che ha la forma di un icosaedro troncato, come un pallone da calcio ha attirato molta attenzione per la perfezione della sua struttura poliedrica, la sua relativa stabilità e gli orizzonti che apre essendo una nuova forma allotropica del carbonio (Fig. 10).

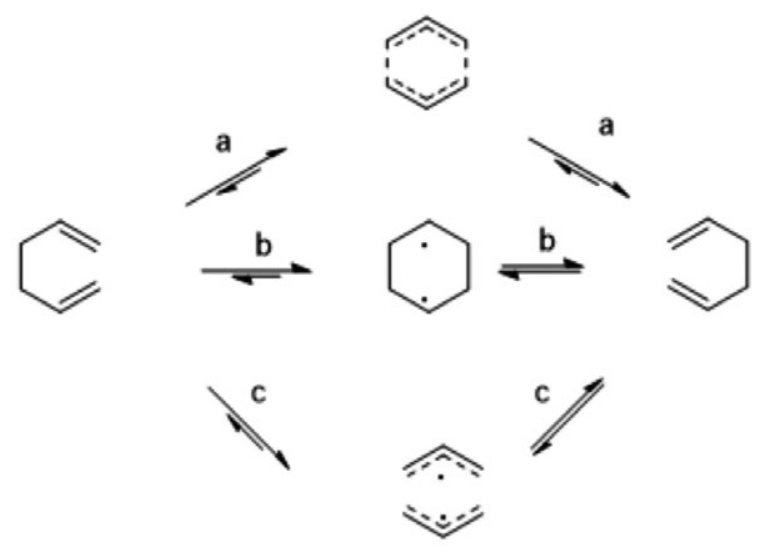

Fig. 10.

Come disse uno degli scopritori del $\mathrm{C}_{60}$, R. Smalley, "Naturalmente, ci devono essere centinaia di meccanismi per i quali si possa andare $\mathrm{al}_{60}$ che coinvolgono forze che portano alla spontanea auto sistemazione (self-assembly) degli atomi di carbonio. Questa affermazione evidenzia la grande complessità del problema e la terribile incompletezza del nostro sapere. Più capiamo delle origini di una reazione, più capiamo che i meccanismi sono intrinsecamente molteplici, e comportano un riarrangiamento geometrico delle posizioni relative dei nuclei che avvengono nelle molecole che interagiscono e si muovono lungo un percorso sulla superficie dell'energia potenziale (PES), traversando creste che costituiscono le barriere nella reazione. Vedere una PES ricorda un paesaggio collinare, e si può continuare la metafora paragonando la riuscita trasformazione della molecola con il successo di un montanaro che si muove dalla valle dei reagenti a quella dei prodotti attraverso uno dei passi più facilmente accessibili. 
Ma i paesaggi collinari del mondo reale, come quelli calcolati, non sono così monotoni da presentare un unico passo tra le valli, cosicché traiettorie che portano a reazione non sono rare e il loro numero cresce rapidamente se si fa crescere di un po' l'energia cinetica, condizione nella quale la necessità di passare tutte per lo stesso punto di sella diviene una caratteristica meno stringente. Inoltre, se gli spostamenti dei nuclei nel corso di un riarrangiamento sono abbastanza piccoli, la reazione può avvenire anche attraverso (sotto) la barriera d'energia, per via di un tunnel quanto meccanico.

Consideriamo ora un fenomeno che si possa spiegare in due modi, l'uno per la via $\mathrm{A}$, che ne è il fattore determinante
(1) $\Pi=\mathrm{A}$

mentre l'altro si può spiegare col contributo di due modi, A e B

(2) $\Pi=c a A+c b B$

Può allora ben essere che nel caso di una osservabile sia valida una spiegazione semplice che spieghi bene $\mathrm{i}$ fatti, e con il rasoio di Occam sarà questa la via preferita. Ma c'è d'aspettarsi che nell'universo che si osserva, ci siano diverse osservabili, $\Pi 1, \Pi 2, \Pi 3$, che presentano una spiegazione più complessa, come nell'eq 2 . Anche quando uno solo di tali modi è presente, questo fatto può portare ad individuare ulteriori esperimenti, e quindi può avere un ruolo propositivo nella ricerca.

Questo approccio potrebbe esser formalizzato con il metodo epistemologico proposto all'inizio del '900 dal geologo di Chicago, TC Chamberlain,[25] e poi usato da J. Platt come la base di un metodo per avere conclusioni rigorose, o che entrambe siano pensabili come gemmazioni.[26] Dal metodo induttivo di F. Bacon, sottolineando che il fatto di ottenere più test simultaneamente sperimentabili, ciascuno con le proprietà di arrivare alle conclusioni deve arrivare a conclusioni più ricche e più complete che l'insistenza di un solo modo. La storia della chimica offre moltissimi esempi di errori provocati dall'uso troppo stretto di ipotesi.

Chi si presenta nelle vesti di un critico del rasoio di Occam può forse esser accusato di assumersi capacità filosofiche non garantite, in quanto si implicherebbe senza dichiaralo la preferenza per un modello semplice e la credenza che viviamo in un universo semplice. Chi però 
continua e dice che il mondo non può esser così semplice e su questa base invalida l'applicazione del rasoio di Occam negli studi scientifici ha ragione?. Ma è poi vero che il credere in un universo semplice è necessariamente implicato dall'essere filosoficamente onesti quado si invoca il rasoio d Occam? Dal punto di vista epistemologico, chi cerca dei mattoni da cui si possa ricostituire l'architettura miracolosamente complessa della natura? è questo l'uso corretto del rasoio di Occam? Non è meglio usare questo strumento per identificare questi moduli individuali, piuttosto che usarlo per l'intera Weltanschaung che si può costruire da essi? Il principio della parsimonia non è un'affermazione metafisica per cui l'universo è come è. Ciascuno sa bene che è estremamente complesso e il rasoio di Occam, che funziona, è una ricetta per capire come il sistema si organizzi. Secondo questo punto di vista prammatico, il rasoio di Occam serve come un principio operativo, non come una regola o legge della natura.

Nel cosiddetto metodo scientifico, si cercano esperimenti che possano falsificare delle affermazioni. La rinuncia a idee che avviene quando un modello non funziona si assume sia completo e irreversibile, purché questo esperimento sia accessibile a criteri di validità intellettuale e l' esecuzione sia competente e pertanto riproducibile

Nell'interpretazione prammatica del rasoio di Occam, non si applica un linguaggio tanto irrevocabile, ma si possono usare due modelli per ogni altro aspetto egualmente validi, e la scelta tra i due ipotesi deve bensì esser presa in favore della più semplice, ma il fatto di scartare quella più complessa deve esser inteso come condizionale. L'idea che è stata lasciata può essere riconsiderata in favore di un'altra ritenuta migliore al momento, e non scartata definitivamente perché la relativa ipotesi potrebbe non superare ulteriori test futuri. Non deve quindi essere indicata come scartata, per aver violato il rasoio di Occam, un'espressione che pertiene a un mondo in cui i test che li escludono sono più rigorosi.

$\mathrm{Ma}$, a parte il senso intuitivo di quello che è giusto, perché si dovrebbe preferire questo modello a quello della retta? Una risposta può venire dai gradi di libertà di ogni modello che funziona. In statisti$\mathrm{ca},[27]$ il numero di gradi di libertà è la differenza tra il numero di osservazioni sperimentali indipendenti e il numero di parametri aggiustabili nelle funzioni che cercano di descrivere la relazione tra y e x. è assiomatico che qualunque funzione con un numero di parametri aggiustabili eguale o maggiore del modello può esser fatto passare esat- 
tamente per i punti $(\mathrm{x}, \mathrm{y})$ nel grafico, ma non è necessariamente vero che una funzione con un minor numero di aggiustabili del numero di osservazioni passi per i vari punti. Se capita che lo faccia, allora la funzione matematica che si è usata come modello ha avuto qualche successo nel descrivere gli avvenimenti che abbiamo studiato sperimentalmente.

I punti in cui passa la funzione, senza alcun motivo algebrico che così esiga, costituisce una misura, che sarà seguita da altre e le previsioni di ciascuna funzione saranno valutate. Ad esempio, si presuma che si cerchi una funzione matematica tra $\mathrm{x}$ e $\mathrm{y}$ che possa predire altri punti futuri sul grafico; si avrà più tendenza a scegliere quei modelli che abbiano avuto maggior successo nel predire le misure che abbiam fatto fino a quel momento. Questo sarà il modello con il maggior numero di gradi di libertà, o quello col numero minore di parametri aggiustabili cioè il più semplice.[16]

La rappresentazione migliore di $\mathrm{y}$ in funzione di $\mathrm{x}$ serve anche a illustrare una qualche finale rappresentazione per applicare il raso di Occam come un metodo operazionale. Il numero di modelli egualmente ben funzionanti in una classe è in genere legato alla complessità di tale classe, ad esempio c'è una sola retta che passi attraverso tutti punti di $(x, y)$, per la quale c'è solo una di quelle relazioni, ma per rappresentare la relazione $\mathrm{x}, \mathrm{y}$ in generale con linee non rette che passino per tutti i punti c'è un'infinità di soluzioni.[28,29] D'altra parte, perchè il numero di parametri richiesto per descrivere line frastagliate necessarie per modelli complessi, occorre un'infinità di linee frastagliate che passino esattamente per tutti i punti. Con le osservazioni fatte fin qui, non c'è nessun metodo difendibile logicamente per scegliere tra uno e infinito.[16]

Il rasoio di Occam, costringe a limitare il numero di ipotesi da considerare e, di conseguenza, riduce il ruolo dell'immaginazione, ma è lo scotto da pagare per evitare di trovarsi di fronte a un numero illimitato di soluzioni. Qualcuno potrebbe chiedersi se gli scienziati pensino di capire tutto su come funziona l'universo. Naturalmente la risposta è assolutamente no. Ma, se vogliamo trarre un qualunque senso dal mondo, dobbiamo semplificare. Se dovessimo operare sotto le condizioni di eguale opportunità, avremo un mondo troppo complesso per essere descritto e una descrizione auto contradittoria della natura che ci impedirebbe di sentir mai che stavamo facendo qualche progresso nel capire o utilizzare l'ambiente.[30,31] 
Abbiamo mai progredito? Con dolore ci rendiamo conto di tutte le ambiguità dell'idea di progresso nel ventesimo secolo, durante il quale la scienza è fiorita, ma altrettanto ha fatto la profonda sfiducia in tale idea da parte di persone ragionevoli.

Se ci distacchiamo dalle implicazioni filosofiche derivate dal trattare il rasoio di Occam come un semplice principio operativo, rischiamo di macchiarci di codardia intellettuale? Crediamo davvero che una correlazione lineare sia la più adatta? $\mathrm{E}$ in verità, se non otteniamo tali valori, non penseremo a un errore nell' esperimento? E questa aspettativa non è una fede in un universo semplice?

\section{CONCLUSIONI}

A modesto parere dell'estensore di questa nota, il divorzio tra le varie discipline è ormai consumato, e, visto che sia le parole sia i simboli vengono usarti in maniera differente non c'è probabilità di tornare a una idilliaca unità, comunque impensabile per la veloce evoluzione delle discipline. Si è cercato sopra di indicare le caratteristiche del linguaggio della chimica, basato sulle formule che molto bene descrivono come sia fatto l'ambiente che ci circonda e come possiamo trarne cose utili. Per altro, già il passare dalle formule dei reagenti a quelle dei prodotti, attraverso le equazioni chimiche causa problemi, in quanto questa relazione è stata a lungo indicata col segno di eguaglianza $(=)$, con un significato ben diverso, tuttavia, da quello che si addotta in matematica (Reagenti=Prodotti). In chimica si poneva l'accento sul significato quantitativo niente si crea, niente si distrugge, ma tutto si trasforma. Ora del tutto abbandonato per i troppi carichi che si erano accumulati su di lui, il segno $=$ è stato sostituito dalla freccia $(\rightarrow)$ che distingue un prius e un post, senza ulteriori complicazioni.

\section{BIBLIOGRAFIA}

1. Luckenbach, R, The Beilstein Handbook of Organic Chemistry, J. Chem. Inf. Comput. Sci. 1981, 21, 82-83.

2. Beilstein Handbuch der organische Chemie, Beilstein Institute for the develpment of chemistry, Frankfurt.

3. Gmelin Handbook of Inorganic and Organometallic Chemistry, Beilstein 
Institute for the develpment of chemistry, Frankfurt.

4. Albini, A, Light, molecules, reactions and health. Elsevier, London, 2020.

5. Holick, MF, Vitamin D, a 3 steps strategy to cure our most common health problems, Hudson Free Press, New York, 2010.

6. Holick, MF, Am J Clin Natur, 2004, 80, 16885-1689.

7. Anfinsen CB ,1973, , Science, 1973, 181, 223-230.

8. Ramachandran GN, Ramakrishnan, C, Sasisekharan, V, Stereochemistry of polypeptide chain configurations". J. Mol. Biol. 7, 95-9.

9. R Krämer, JM Lehn, AM Marguis/Rigault, , Proc Natl Acad Sci USA, 1993, 90, 5394-5398.

10. Kim YE, Hipp MS, Bracher A, Hayer-Hartl M, Hartl, FU, Annu Rev Biochem, 2013, 82, 323-355.

11. Bryngelson JD, Onuchic JN, Socci ND, Wolynes PG, Proteins,1995, 21, 167-95.

12. Compiani M, Capriotti, E (2013). "Computational and theoretical methods for protein folding”. Biochemistry. 52 (48): 8601-24.

13. Fersht, AR, Proceedings of the National Academy of Sciences of the United States of America, 2000, 97, 1525-1529.

14. Bryngelson, JD, Wlynes,P, J Phys Chem, 1989, 93, 6902-6915.

15. Hargrave, PA, Dowell, JH, Rhodopsin and phototransuction, Int. rev. Cytol, 37b, 1992, 9-97.

16. Hoffmann, R, Minkin, VI, Carpenter, BK, 1997, HYLE-International Journal for Philosophy of Chemistry, 3 (1997), 1-28.

17. Muller, A, Bulletin de Société Chimique, Paris, 1886, 45, 438.

18. W. Hückel, W, Theoretische Grundlagen der Organischen Chemie, Akademische Verlagsgesellschaft, Leipzig, 1934.

19. Wheland, GW, Advanced Organic Chemistry, Wiley, New York, 1960.

20. Rice, F,. Teller, Journal of Chemical Physics, 1936, 489.

21. R.B. Woodward and R. Hoffmann, "The Conservation of Orbital Symmetry, Verlag Chemie, Weinheim, 1970.

22. Bhunia, A, Rot, t, Pachfule, P, Rajamohanan, PR, Biju, AT, Angew. Chem, IEE, 2013, 52, 10224-10227.

23. Hammond, G, DeBoer, C, J. Am. Chem. Soc, 1963, 86, 899-902.

24. Kroto, HW, Heath, JR, O’Brien, SC, Curl, RF, Smalley, RE, Nature, 1985, 6042. 162-163.

25. Chaberlain, TC, The method of multiple working hypotheses, Studies for students, 1897, 837-848.

26. Platt, JJ, Science, 1964,146, 4.

27. Schuster, HG, Deterministic Chaos, Verlag Chemie, Weinheim, 1984.

28. Kalinowski, K, Tadeusz, Krygowski, TM, Croatica Chim Acta, 1988, 58, 107-126.

29. Rychlewski, J, Journal of Applied Mathematics and Mechanics, 1984. 48, 303-314.

30. Jeffreys, WH, Theory of Probability, Oxford University, Oxford, 1939.

31. Jefferys, WH, Berger, American Scientist, 1992, 80 64-72. 
